\title{
Propeller Flaps in the Head and Neck
}

\author{
Adriana Cordova, MD, $\mathrm{PhD}^{1}$ Salvatore D’Arpa, MD, $\mathrm{PhD}^{1}$ Fernando Rosatti, MD ${ }^{1}$ \\ Marta Nichelini, MD ${ }^{1}$ Giovanni Maria D’Antonio, MD ${ }^{1}$ Salvatore Giordano, MD, PhD, FACS ${ }^{1}$ \\ Francesca Toia, MD, $\mathrm{PhD}^{1}$
}

${ }^{1}$ Division of Plastic and Reconstructive Surgery, Department of Surgical, Oncological and Oral Sciences, University of Palermo, Palermo, Italy

Semin Plast Surg 2020;34:165-170.

\begin{abstract}
Address for correspondence Francesca Toia, MD, PhD, Division of Plastic and Reconstructive Surgery, Department of Surgical, Oncological and Oral Sciences, University of Palermo, Via del Vespro, Palermo 129. 90127, Italy (e-mail: francesca.toia@unipa.it).
\end{abstract}

\author{
Abstract \\ Keywords \\ - head and neck \\ reconstruction \\ - perforator flaps \\ - propeller flaps \\ - facial artery \\ perforator \\ - supratrochlear artery \\ perforator \\ - supraclavicular artery \\ perforator \\ - lingual flaps
}

Propeller flaps have significantly expanded the reconstruction possibilities in the head and neck region. They allow for increased flap mobility and better scar concealing, and/or to perform a one-stage reconstruction with local tissue of similar color and texture, where multiple surgeries would be needed with traditional flaps or even free flaps would be required. This article describes the main propeller flaps for one-stage reconstruction in the head-neck region (facial artery perforator, supratrochlear artery axial perforator, deep lingual artery axial perforator, and anterior supraclavicular artery perforator flaps), their indications, and possible complications. Aesthetic and functional results of propeller flaps in the head and neck region are very good and the complication rate is low, but due to their surgical complexity and the availability of many simpler local flaps, they are indicated only in select cases where local flaps are unavailable or would require multiple staged procedures to complete the construction.
The head and neck area is a highly vascularized region, similar in vessel density only to that of the perineal area. For this reason, a multitude of flaps can be designed based on perforator or axial vessels. Propeller flaps are a versatile and safe option that allows reconstruction of a large variety of defects in the head and neck region due to an increased mobility when compared with other local flaps. They can be based on different types of pedicles that can be classified, according to the Tokyo consensus and following update, ${ }^{1,2}$ as follows:

1. Subcutaneous pedicled propeller flaps.

2. Perforator pedicled propeller flaps.

3. Supercharged pedicle propeller flaps.

4. Axial pedicled propeller flaps.

This review is intended to describe the main propeller flaps for head and neck reconstruction and discuss their indications on the basis of the defect location. Specifically, the following propeller flaps will be described:

1. Perforator propeller flaps based on the facial artery for reconstruction of the perioral region and lateral nose.
2. Axial propeller flaps based on the supratrochlear artery for reconstruction of nasal and periorbital soft-tissue defects.

3. Axial propeller flaps based on the deep lingual artery for the reconstruction of intraoral defects.

4. Perforator supraclavicular propeller flaps based on the supraclavicular artery for reconstruction of the head and neck region.

\section{Facial Artery Perforator Propeller Flap}

The facial artery is the main arterial branch to the skin of the face; it courses from the midpoint of the horizontal branch of the jaw to the internal canthus where it becomes the lateral nasal artery. Around 100 perforating branches originate from its trunk, allowing the design of a great variety of free-style propeller flaps, mostly used for the reconstruction of the alar region of the nose and the perioral region, especially for the upper hemi-lip aesthetic subunit.

Facial artery perforator flaps help reconstruct facial defects in a single stage while using tissue that matches the color and texture of the face. ${ }^{3}$ In selected cases, the reconstruction with a
Issue Theme Propeller Flaps; Guest editors: Geoffrey G. Hallock, MD, Marco Pignatti, MD
Copyright (C) 2020 by Thieme Medical Publishers, Inc., 333 Seventh Avenue, New York, NY 10001, USA. Tel: +1(212) 760-0888. 


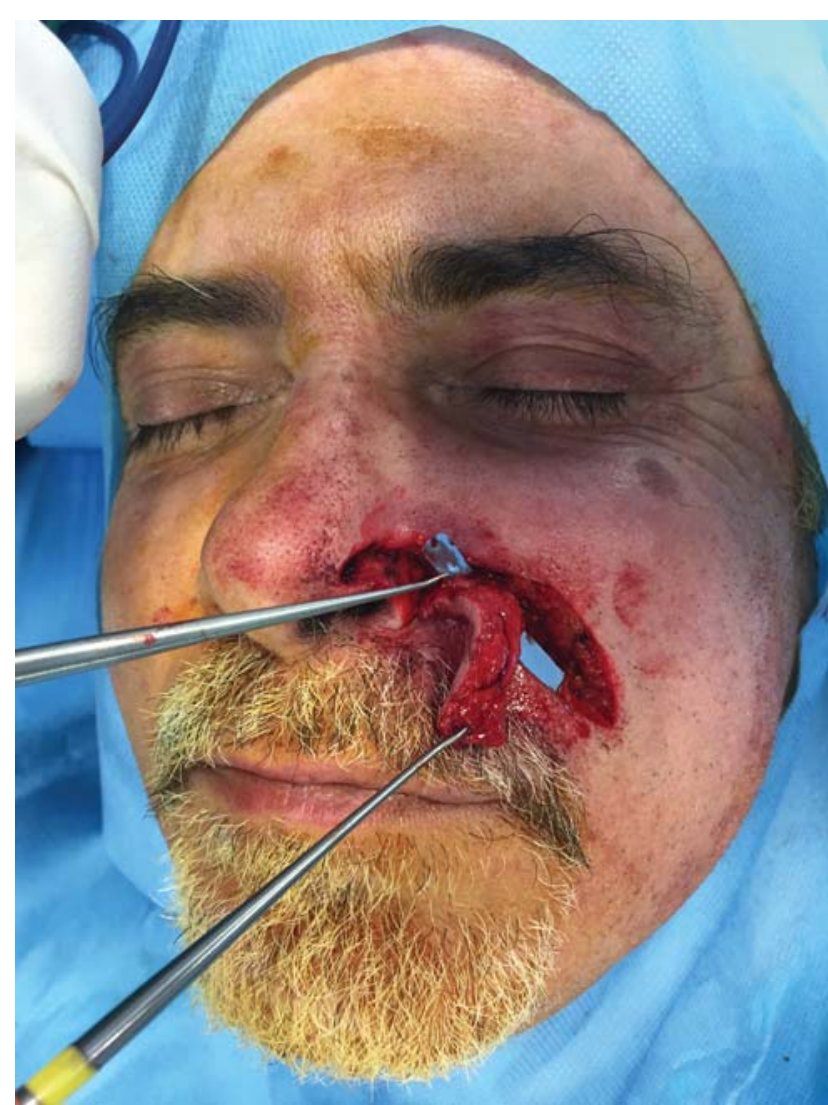

Fig. 1 Intraoperative view of a facial perforator flap for nasal ala reconstruction. The pivot point corresponds to the pedicle only, and the flap rotates $\sim 180$ degrees to reach the defect.

facial artery perforator propeller (FAPP) flap has some advantages compared with other local flaps: that is, the possibility of repairing adjacent facial esthetic units in a single surgical procedure and the primary closure of the donor area by hiding the scar within the mesolabial fold. ${ }^{4}$ Single-stage transfer does not impair the possibility of performing secondary revisions to improve the eventual aesthetic result.

\section{Indications}

The FAPP flaps are mainly indicated for the reconstruction of nasal ala or perioral defects.

\section{Nasal Ala Defects}

Since its first description in 1840 , the nasolabial flap is still one of the most frequently used techniques for nose reconstruction, although it has undergone several modifications over time. The nasolabial flap provides skin of similar texture and color, while allowing primary closure of the donor site leaving a scar hidden in a natural fold. Due to the usual laxity of cheek tissues, there is enough tissue to reconstruct the whole ala. The proximity to the defect and the advantage of hiding the scar in the nasal grove make this flap a valid alternative to the forehead flap.

The introduction of the nasolabial propeller flap allows a one-stage reconstruction, overcoming the main drawback of the classical nasolabial interpolation flap, which traditionally requires two surgical stages., 5

Thanks to the free-style and propeller design concepts, once a perforator vessel is identified, the nasolabial flap can be rotated up to 180 degrees. The pivot point of the flap corresponds to the perforating vessels only, achieving a significantly increased mobility when compared with other local cutaneous or subcutaneous pedicle flaps ( - Fig. 1). For this reason, distortions and cosmetic defects due to excessive rotation and pedicle bulk are not generally observed with this flap. ${ }^{6}$

\section{Perioral Defects}

Facial artery perforator flaps are also superior to traditional nasolabial island flaps for the reconstruction of perioral defects (-Fig. 2). Traditional nasolabial island flaps cannot cover defects of the philtrum; so, a second flap is required for a large defect involving the philtrum. Facial artery perforator flaps can instead allow reconstruction of large defects with a single flap with better scar concealing as the donor site can be directly closed.

In their anatomic study, Hofer et al identified a mean of 5.7 adequate facial artery perforators with a direct path from the skin to the facial artery (range: $3-9$ ) for the reconstruction of perioral defects. ${ }^{7}$ The presence of these many perforators enables the harvest of a tailor-made flap of the same thickness of the defect, with increased mobility due to rotation up to 180 degrees, and all without the need for a second-stage reconstruction. ${ }^{7}$
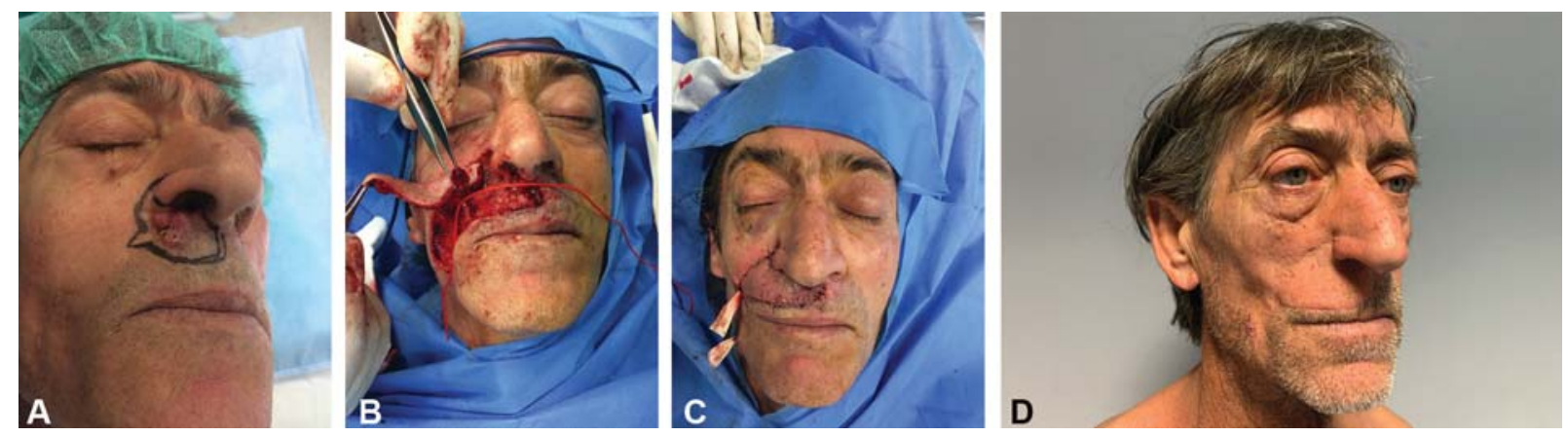

Fig. 2 Reconstruction of the upper lip with a facial artery perforator propeller flap. (A) Male with a nodular basal cell carcinoma of the upper lip encroaching on the nose that has been marked for excision. (B) Intraoperative view after tumor resection. Flap has been circumferentially incised and remains attached only by its eccentrically located vascular pedicle. (C) Flap has been inset after counterclockwise rotation about its perforator. (D) One year postoperative result. 


\section{Surgical Technique}

Using an 8-MHz Doppler, perforating vessels of the facial artery are preoperatively identified and marked, and then the flap is designed accordingly. Most of the time, the course of the facial artery is confined within $5 \mathrm{~mm}$ from the nasolabial fold. The most constantly encountered perforator is present lateral and slightly inferior to the oral commissure. ${ }^{8}$ Another relevant anatomical reference point for facial artery perforators is located approximately $1.5 \mathrm{~cm}$ lateral to the oral commissure, at the same level. ${ }^{9}$

After an exploratory incision, the most suitable perforator is chosen. During the skeletonization of that perforating vessel, any connection with the surrounding tissue must be interrupted, to avoid any restriction or twisting of the pedicle, which may cause collapse of the vessel. Any traction on the perforating vessel must be avoided, as the vessel may react with vasospasm or the intima may ultimately break, with an increased thrombosis risk. The longer the pedicle, the more widely the torsion forces are distributed and the less the risk of twisting and occlusion. While arterial problems are rare, venous congestion is more likely to occur with twisting of the pedicle.

Once the best perforator closest to the defect has been chosen and isolated, the flap can be eventually replanned based on the defect and then harvested. The propeller movement allows flap rotation up to 180 degrees. The direction of rotation is chosen by rotating the flap clockwise and counterclockwise to verify the most convenient option before definitively insetting the flap. Color, capillary refilling, and bleeding of the flap are evaluated after a few minutes. In case of eventually excessive venous congestion, the flap is derotated for a few days. Mild venous congestion, however, is well tolerated by these robust flaps that may develop additional outflow connections early postoperatively to solve the venous congestion without evolving into necrosis. The final step is closure of the donor site (within the nasolabial fold) by direct suturing. ${ }^{10}$

\section{Supratrochlear Artery Axial Propeller Flap}

The supratrochlear artery axial propeller (STAAP) flap is a variation of the supratrochlear artery flap, which is one of the oldest axial flaps described (it was already in use in the 6th century BC) and is still today considered the gold standard in the reconstruction of nose defects. ${ }^{11}$ Based on the recent advances in perforator flaps, our group has applied the propeller concept also to this flap, describing the STAAP flap for a onestage nasal reconstruction. The second stage of pedicle division and brow reconstruction may be avoided. The technique, however, does not restrict the possibility of reconstructing the eyebrow and revising the flap for aesthetic improvements should the patient and surgeon want it.

The supratrochlear artery emerges from the superomedial portion of the orbital margin approximately 1.7 to $2.2 \mathrm{~cm}$ from the midline of the forehead and continues its course in a paramedian position approximately $2.0 \mathrm{~cm}$ from the midline. ${ }^{12}$ The supratrochlear artery first travels in a deeper plane, passing under the orbicularis oculi muscle then through the corrugator supercilii muscle, and then go superficially $3 \mathrm{~cm}$ from the upper orbital border in a subcutaneous plane.

The STAAP flap associates the design of a traditional forehead flap with the "perforator-like" dissection of the pedicle and the "propeller rotation" of the skin island, combining the advantages of a classic forehead flap with those of a one-stage reconstruction, without an increase in complication rates, even in patients with comorbidities. ${ }^{13}$

\section{Indications}

The STAAP flap has been described for one-stage nasal reconstruction, but its use has been extended for the reconstruction of the infraorbital region (-Fig. 3). ${ }^{13,14}$ However, unlike the median forehead flap, the STAAP is not indicated in the reconstruction of distal nasal aesthetic subunits (nasal tip or nasal wing), as it requires continuity of the defect with the pivot point of the flap, but rather is a good solution for the reconstruction of the entire pyramid or defects of the dorsum of the nose.

\section{Surgical Technique}

The STAAP vascular pedicle most often arises from the orbital rim 1 to $2 \mathrm{~cm}$ lateral to the midline. It is more commonly located lateral to the medial canthus in males and medial to it in females. ${ }^{15}$ Using an 8-MHz Doppler, the exact point of emergency of the supratrochlear vessels can be marked preoperatively. The desired flap is designed about this point.

Elevation begins as a classic forehead flap until the supratrochlear artery is isolated, and the flap is completely islanded incising the skin at the eyebrow margin over the supraorbital foramen. The supratrochlear artery is then isolated dissecting it down to its emergence from the frontal bone. Intramuscular pedicle dissection can be performed using Stevens' scissors and a self-retaining retractor. A vessel loop can be applied around the pedicle to allow for gentle traction and facilitate dissection.

Once finished, the flap can be rotated up to 180 degrees clockwise or counterclockwise, choosing the least cumbersome direction of rotation. The caudal part of the flap can be used to partially cover the donor area, reducing the risk of pedicle compression and maintaining a satisfactory intereyebrow distance. If possible, the remaining donor defect is closed directly. If that is too large, any residual open area can be left to heal by secondary intention, with a skin graft, or regenerative dermis. ${ }^{10}$

Necrosis of the distal end of the flap can occur postoperatively, but it is a rare event ( $1 / 25$ patients in our previously published series). ${ }^{14}$

\section{Deep Lingual Artery Axial Propeller Flap}

The deep lingual artery axial propeller (DLAAP) flap is based on the deep lingual artery and is indicated for medium to large size intraoral defects as an initial reconstructive option or salvage of other flap failures. ${ }^{16,17}$ Lingual flaps represent an effective option for intraoral reconstruction with local mucosa. The traditional technique is rarely used because it requires two surgical stages, causing greater patient discomfort during flap delay. 

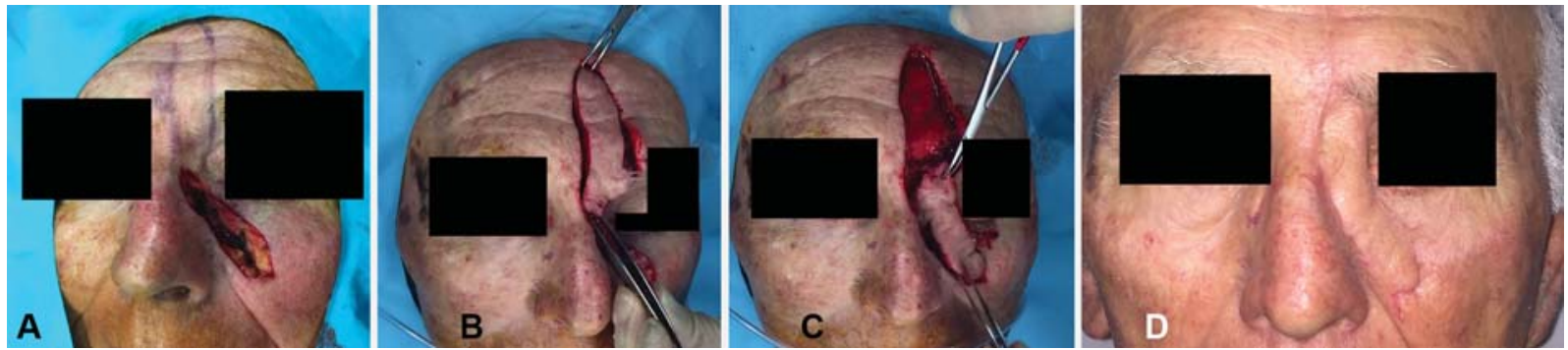

Fig. 3 Infraorbital reconstruction with a supratrochlear artery axial perforator flap after resection of a basal cell carcinoma. (A) Vertical design of the flap based on the ipsilateral supratrochlear vessels. (B) Flap dissection has been completed, (C) then rotated clockwise 170 degrees into the defect. (D) Six months postoperative result. Note that the donor area was closed by primary intention.

The lingual artery enters the tongue and runs between the hyoglossus and genioglossus muscles. Its terminal part, known as the deep lingual artery, has a tortuous course along the ventral tongue surface and runs toward the tip of the tongue where it becomes superficial to be covered only by mucosa. ${ }^{18}$

In 2015, our group described the application of the perforator and propeller technique to this oral mucosal area for the first time, introducing the DLAAP flap as a modification of the traditional tongue flap. ${ }^{16}$ The DLAAP flap as such has an increased arc of rotation as can be rotated any angle up to 180 degrees to allow a one-stage intraoral reconstruction with local tissue.

The pliability of the soft tissue around the DLAAP flap pedicle contributes to the increased flap mobility. ${ }^{19-21}$ Doubtless, the rich mucosal vascularization and the laxity of the soft tissue surrounding the pedicle increase the survival rate of these flaps. The typical villous appearance of the tongue tissue disappears once the flap is transferred to the recipient site, becoming as smooth as the surrounding mucosa within 1 month after surgery. ${ }^{6}$ When compared with reconstruction with other local free flaps, the DLAAP flap has proven to have a quicker functional recovery and satisfactory outcome with oral function not being impaired after flap harvesting, as highlighted by 6 - and 12-month postoperative Functional Intraoral Glasgow Scale (FIGS) rate. ${ }^{21,22}$

\section{Indications}

As mentioned, the DLAAP flap may be indicated as the first or salvage option for a one-stage reconstruction of medium defects of the mucosal cheek, retromolar trigone, and the hard and soft palate (-Fig. 4). The floor of the mouth can also be repaired, using it as advancement flap, except for defects requiring a pullthrough technique, which otherwise would need bulkier flaps.

\section{Surgical Technique}

The flap is elliptically shaped and taken from the lateral side of the tongue. The dimensions will depend on the size of the tongue and of the defect, with a median length of approximately $6 \mathrm{~cm}$. Although the vascular anatomy of the tongue is constant, preoperative localization of the deep lingual artery using an audible Doppler is useful and possible when the patient is relaxed under anesthesia. The presence of a welldeveloped venous plexus with a larger caliber is usually associated with the artery, and also can be easily harvested between the anterior two-thirds and the posterior third of the tongue. ${ }^{16,19}$

Elevation of the DLAAP flap usually starts from the anterior portion of the tongue with electrocautery, and then muscle dissection is performed using Stevens' scissors in the posterior third. A vessel loop around the pedicle can be useful to allow gentle traction and facilitate dissection. Once the vascular pedicle has been sufficiently dissected, the flap can be circumferentially incised and rotated as a propeller on multiple planes to reach the defect. In the oral cavity, differently from other body regions, the flap needs to rotate on a combination of axial, coronal, and sagittal planes depending on the exact defect location. The lingual donor site is finally closed primarily. ${ }^{16}$
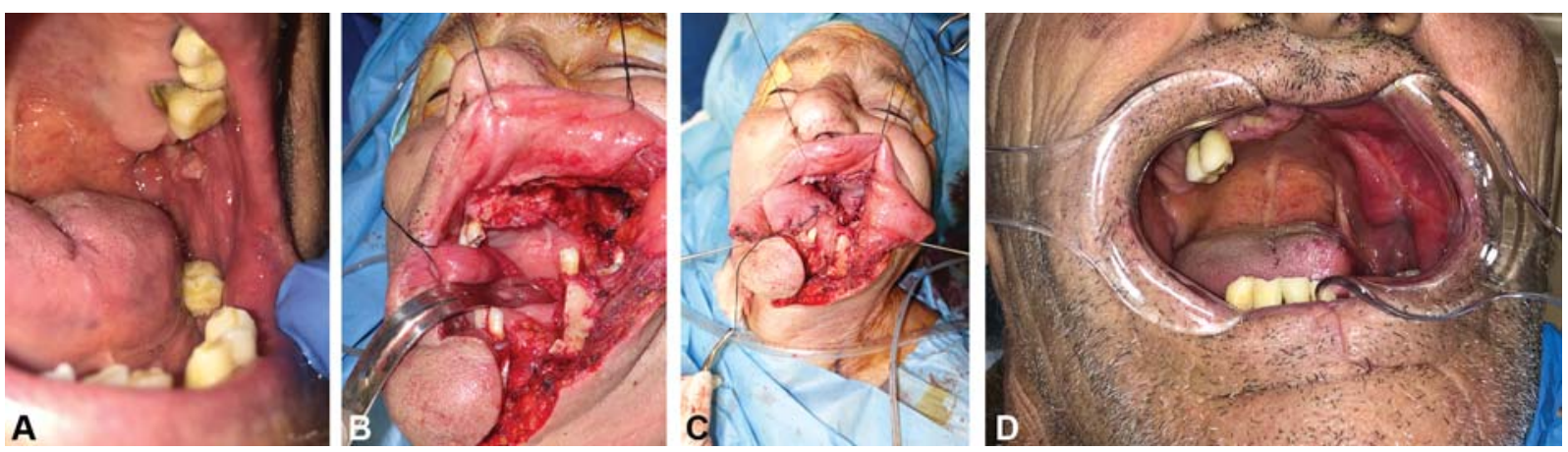

Fig. 4 Reconstruction of the palate with a deep lingual artery axial propeller (DLAAP) flap. (A) A 70-year-old male with a squamocellular cancer infiltrating the upper arch of left gingival mucosa. (B) Complete tumor extirpation resulted in a defect involving the left upper alveolar ridge and destruction of the maxillary sinus floor. (C) After filling of the maxillary sinus with a Bichat's fat pad flap, the mucosal defect was reconstructed with a DLAAP flap harvested from the ipsilateral tongue. (D) Excellent restoration of mucosa seen 2 months later. 


\section{Supraclavicular Artery Propeller Flap}

The anterior supraclavicular artery perforator (a-SAP) flap, described by Pallua and Wolter, ${ }^{23,24}$ is a variation of the supraclavicular island flap based instead on perforator and propeller concepts. The a-SAP flap is vascularized by the supraclavicular artery as described by Cormack and Lamberty, ${ }^{25}$ more precisely by its anterior branch that runs along the deltopectoral groove after originating from the transverse cervical artery. Although different flaps based on the supraclavicular artery have been described, ${ }^{25-28}$ the a-SAP flap allows for better flap mobility and scar concealment. This is a versatile option for the reconstruction of head and neck defects with local tissue of similar texture and color. Moreover, it can be longer than the classical flap, as it can reach $35 \mathrm{~cm}$ of length.

\section{Indications}

The pedicled a-SAP flap is indicated for neck and face reconstruction, particularly following burns or tumor resection, as the donor site is outside the usual zone of injury, but tissue color and characteristics are similar. The flap can also sometimes be utilized for intraoral and pharyngeal reconstruction.

\section{Surgical Technique}

The a-SAP flap can be designed up to $35 \mathrm{~cm}$ in length and up to $5 \mathrm{~cm}$ in width while still allowing direct donor site closure. The pedicle is identified with an audible Doppler at the level of the clavicle. Then the flap is raised in a suprafascial plane up to the clavicle, where the anterior branch is identified and dissected up to the transverse cervical artery. At that point, several lymph nodes can be encountered and can be spared. If a periosteocutaneous flap is needed, the periosteum of the clavicle can be included in the flap. To decrease the risk of venous congestion, the cephalic vein can also be included with the flap. Once the pedicle has been satisfactorily dissected, the flap can be rotated on that vascular pivot point up to 180 degrees to reach the neck or face.

\section{Discussion}

Propeller flaps in the head and neck region are easier and safer compared with propeller flaps from other anatomical areas, particularly in the lower limbs, due their favorable vascular anatomy.

Given the high vascularization of the head and neck region, performing reconstruction with random flaps is very safe; so, the use of a propeller flap has to be justified by having remarkable advantages over traditional local or even free flaps.

Some anatomical considerations about the head and neck region that must be acknowledged are as follows:

- The face is a small area covering approximately $4 \%$ of the body surface; so, facial flaps will be small themselves.

- Vascularity here has the highest density in the body.

- The distribution of facial vessels is anatomically very constant.
- Facial skin can be very loose in elderly patients, which readily allows larger flaps while still permitting primary donor site closure.

- Most facial propeller flaps are axial flaps and only FAPP flaps are free style perforator flaps.

This review has been restricted to the most popular propeller flaps of the head and neck region on the basis of its most important arteries. The main advantage of these propeller flaps is the ability to perform any reconstruction as a one-staged procedure. Harvesting of all requires similar precautions as in other body regions relying on preoperative assurance of the presence of a perforator using the audible Doppler or other modality, identifying intraoperatively a valid perforator, altering the flap design when indicated, verifying the course of the perforator within the flap, and determining the best rotation prior to final insetting of the flap.

In the head and neck region, the higher vascularity and lower flap size reduce the risk of arterial complications. However, there are only few studies about facial perforasomes and their interconnections. ${ }^{29,30}$

FAPP flaps are free style flaps, and they are used mostly for the distal nasal defects and perioral reconstruction. Axial propeller flaps like STAAP, DLAAP, and SAP have a wellknown anatomy which helps flap design and harvesting.

Within the oral cavity, propeller flaps harvesting is similar to skin flap harvesting. Here, externally propeller flaps reduce the necessary surgical time and perform a likewith-like reconstruction, inevitably decreasing the need for microsurgery.

Propeller flaps in the head, neck, and oral cavity are usually of small size but highly vascularized; yet, the greatest complication risk is venous congestion. To reduce that, it is very important to fully dissect the pedicle avoiding any tension, and to avoid hematoma in the postoperative period. A very important surgical tip is to meticulously check flap tension upon pedicle rotation, which can lead to venous congestion, which is the most common cause of facial axial propeller flap failure. ${ }^{21}$ Venous congestion is usually due to a mechanical compression, hematoma, or excessive pedicle tension. In the third case, some stich removal can help improve the venous drainage. However, venous congestion not always involves the whole flap but can be partial to result in superficial epidermolysis, which heals uneventfully.

Head and neck propeller flaps have lower complication rates than other body regions, but because of their surgical complexity and the availability of many simpler local flaps, they are indicated only in selected cases where local flaps are unavailable or require several staged procedures to complete the desired reconstruction.

Funding

None.

Conflict of Interest

None declared. 


\section{References}

1 Pignatti M, Ogawa R, Hallock GG, et al. The "Tokyo" consensus on propeller flaps. Plast Reconstr Surg 2011;127(02):716-722

2 Toia F, D'Arpa S, Pignatti M, Noel W, Cordova A. Axial propeller flaps: a proposal for update of the "Tokyo consensus on propeller flaps". J Plast Reconstr Aesthet Surg 2017;70(06):857-860

3 Lee JY, Kim JM, Kwon H, Jung SN, Shim HS, Kim SW. Freestyle local perforator flaps for facial reconstruction. BioMed Res Int 2015; 2015:707065

4 Ruiz-Moya A, Lagares-Borrego A, Infante-Cossío P. Propeller facial artery perforator flap as first reconstructive option for nasolabial and perinasal complex defects. J Plast Reconstr Aesthet Surg 2015;68(04):457-463

5 D'Arpa S, Pirrello R, Toia F, Moschella F, Cordova A. Reconstruction of nasal alar defects with freestyle facial artery perforator flaps. Facial Plast Surg 2014;30(03):277-286

6 D'Arpa S, Cordova A, Pirrello R, Moschella F. Free style facial artery perforator flap for one stage reconstruction of the nasal ala. J Plast Reconstr Aesthet Surg 2009;62(01):36-42

7 Hofer SOP, Posch NA, Smit X. The facial artery perforator flap for reconstruction of perioral defects. Plast Reconstr Surg 2005;115 (04):996-1003, discussion 1004-1005

$8 \mathrm{Ng}$ ZY, Fogg QA, Shoaib T. Where to find facial artery perforators: a reference point. J Plast Reconstr Aesthet Surg 2010;63(12): 2046-2051

9 Qassemyar Q, Havet E, Sinna R. Vascular basis of the facial artery perforator flap: analysis of 101 perforator territories. Plast Reconstr Surg 2012;129(02):421-429

10 Rossi M, RoggioT, Cajozzo M. Lembi perforanti, propeller e freestyle nella ricostruzione labiale. In: Manuale di Microchirurgia: Lembi perforanti, propeller e freestyle peduncolati. Palermo University Press2017:102-109

11 Menick FJ. Nasal reconstruction with a forehead flap. Clin Plast Surg 2009;36(03):443-459

12 Shumrick KA, Smith TL. The anatomic basis for the design of forehead flaps in nasal reconstruction. Arch Otolaryngol Head Neck Surg 1992;118(04):373-379

13 Cordova A, D’Arpa S, Moschella F. A new one-stage method for nose reconstruction: the supratrochlear artery perforator propeller flap. Plast Reconstr Surg 2012;129(03):571e-573e

14 Cordova A, D’Arpa S, Massimiliano T, Toia F, Moschella F. A propeller flap for single-stage nose reconstruction in selected patients: supratrochlear artery axial propeller flap. Facial Plast Surg 2014;30(03):332-341

15 Ugur MB, Savranlar A, Uzun L, Küçüker H, Cinar F. A reliable surface landmark for localizing supratrochlear artery: medial canthus. Otolaryngol Head Neck Surg 2008;138(02):162-165
16 Santecchia L. Anatomia vascolare della faccia. In: Manuale di Microchirurgia: Lembi Perforanti, Propeller e Freestyle Peduncolati. Palermo University Press; 2017:54-59

17 Cordova A, Toia F, D’Arpa S, Giunta G, Moschella F. A new mucosal propeller flap (deep lingual artery axial propeller): the renaissance of lingual flaps. Plast Reconstr Surg 2015;135(03):584e-594e

18 Hyakusoku H, YamamotoT, Fumiiri M. The propeller flap method. Br J Plast Surg 1991;44(01):53-54

19 Cohen DS, Low GMI, Melkane AE, et al. Establishing a danger zone: an anatomic study of the lingual artery in base of tongue surgery. Laryngoscope 2017;127(01):110-115

20 Squaquara R, Kim Evans KF, Spanio di Spilimbergo S, Mardini S. Intraoral reconstruction using local and regional flaps. Semin Plast Surg 2010;24(02):198-211

21 D'Arpa S, Toia F, Pirrello R, Moschella F, Cordova A. Propeller flaps: a review of indications, technique, and results. BioMed Res Int 2014;2014:986829

22 Goldie SJ, Jackson MS, Soutar DS, Shaw-Dunn J. The functional intraoral Glasgow scale (FIGS) in retromolar trigone cancer patients. J Plast Reconstr Aesthet Surg 2006;59(07):743-746

23 Di Fede V, Grassi R, Toia F, Di Rosa L, Cordova A. FLiGS score: a new method of outcome assessment for lip carcinoma-treated patients. Plast Reconstr Surg Glob Open 2015;3(03):e345

24 Pallua N, Wolter TP. Moving forwards: the anterior supraclavicular artery perforator (a-SAP) flap: a new pedicled or free perforator flap based on the anterior supraclavicular vessels. J Plast Reconstr Aesthet Surg 2013;66:489-496

25 Cormack GC, Lamberty BG, eds. The arterial anatomy of skin flaps. In: Head and Neck. London: Churchill Livingstone; 1986: 419-421

26 Cordova A, Pirrello R, D’Arpa S, et al. Vascular anatomy of the supraclavicular area revisited: feasibility of the free supraclavicular perforator flap. Plast Reconstr Surg 2008;122:1399

27 Ma X, Zheng Y, Xia W, et al. An anatomical study with clinical application of one branch of the supraclavicular artery. Clin Anat 2009;22:215-220

28 Vinh VQ, Van Anh T, Ogawa R, Hyakusoku H. Anatomical and clinical studies of the supraclavicular flap: analysis of 103 flaps used to reconstruct neck scar contractures. Plast Reconstr Surg 2009; 123:1471-1480

29 Lecours C, Saint-Cyr M, Wong C, et al. Freestyle pedicle perforator flaps: clinical results and vascular anatomy. Plast Reconstr Surg 2010;126(05):1589-1603 [Erratum in: Plast Reconstr Surg 2011;127(4):1753]

30 Saint-Cyr M, Wong C, Schaverien M, Mojallal A, Rohrich RJ. The perforasome theory: vascular anatomy and clinical implications. Plast Reconstr Surg 2009;124(05):1529-1544 